\title{
Electrostatic modeling of peptides derived from the V3-loop of HIV-1 gp120: Implications of the interaction with chemokine receptor CCR5
}

\author{
DIMITRIOS MORIKIS ${ }^{1}$, APOSTOLOS K. RIZOS ${ }^{2}$, DEMETRIOS A. SPANDIDOS ${ }^{3}$ and ELIAS KRAMBOVITIS A $^{4,5}$ \\ ${ }^{1}$ Department of Bioengineering, University of California, Riverside, CA 92521, USA; ${ }^{2}$ Department of Chemistry, \\ University of Crete, and Foundation for Research and Technology-Hellas (FORTH); ${ }^{3}$ Department of Virology, \\ Medical School, University of Crete; ${ }^{4}$ Department of Applied Biochemistry and Immunology, Institute of \\ Molecular Biology and Biotechnology, FORTH, Heraklion, Crete; ${ }^{5}$ Department of Veterinary Medicine, \\ School of Medical Sciences, University of Thessaly, Karditsa, Thessaly, Greece
}

Received December 6, 2006; Accepted January 4, 2007

\begin{abstract}
Infection of $\mathrm{CD}^{+} \mathrm{T}$ cells by macrophage-tropic HIV-1 strains involves interaction of viral gp120 with the host cell chemokine receptor CCR5. The principle neutralizing determinant (PND) of the V3-loop of the HIV-1 gp120 was investigated for its interaction with CCR5 by computational modeling methods at atomic resolution and electrostatic calculations to complement experimental findings. The study focused on the recognition step and examined possible peptidepeptide interactions between various PND-derived peptides from the V3-loop and the N-terminal (Nt) domain of CCR5. These recognition interactions are possible because of the complementary character of the spatial distribution of the predominantly positive electrostatic potentials of the PNDderived peptides and the predominantly negative electrostatic potential of the CCR5Nt domain. The CCR5Nt appears more amenable to interaction with the V3 peptides, than the other CCR5 extracellular domains (ECL), because of its length and the domination of its negative electrostatic potential. On the contrary, ECL2 possesses a predominantly positive electro-
\end{abstract}

Correspondence to: Professor Dimitrios Morikis, Department of Bioengineering, University of California, Riverside, CA 92521, USA E-mail: dmorikis@engr.ucr.edu

Professor Elias Krambovitis, Department of Applied Biochemistry and Immunology, Institute of Molecular Biology and Biotechnology, FORTH, Heraklion, Crete, Greece

E-mail:krambo@imbb.forth.gr

Abbreviations: HIV, human immunodeficiency virus; gp120, viral envelope glycoprotein 120; V3-loop, third variable loop; CCR5, C-C chemokine receptor 5; PND, principle neutralizing determinant; PBL, peripheral blood lymphocytes; Nt, N-terminal; ECL, extracellular loop domain

Key words: HIV-1, gp120, V3-loop, CCR5, Poisson-Boltzmann, electrostatics static potential. There are positive patches in $\mathrm{Nt}$ and negative patches in ECL2, which, following the non-specific recognition of the V3-loop by CCR5 and with the expected local structural rearrangements to facilitate specific binding, may be contributing to the stabilization of the complex. A sequential two-step specific binding, involving different extracellular domains, is conceivable. Although the electrostatic potentials may play a role in a V3-CCR5 interaction, a more specific model cannot be derived in the absence of a threedimensional structure of a gp120/CD4/CCR5 complex.

\section{Introduction}

Monocyte-derived macrophages and $\mathrm{CD}^{+} \mathrm{T}$ lymphocytes are among the primary targets of HIV-1. Increased apoptosis and depletion of uninfected $\mathrm{CD} 4^{+} \mathrm{T}$ lymphocytes during the asymptomatic phase are believed to be the main route of HIV-1 pathogenesis that consequently leads to AIDS (1-4). The viral envelope glycoprotein gp120 has the ability to bind to host cell CD4 and also to interact with the chemokine receptors CCR5 and CXCR4, which are the principle coreceptors for viral entry (5). Deregulation of the immune system via coreceptor usage that leads to activation-induced cell death is an issue that remains to be resolved (6). The third variable region (V3) of the gp120 that forms a loop (V3-loop), the most critical determinant for HIV phenotypic cellular tropism, interacts with these chemokine receptors. We have shown in immunological studies that the semi-conserved tip of V3, also referred to as the principle neutralizing determinant (PND), presented on the surface of macrophages delivers a CCR5mediated activation-induced cell death signal to effector/ memory $\mathrm{CD}^{+} \mathrm{T}$ lymphocytes during the process of antigen presentation (7-9). The role of $\mathrm{V} 3$ is, therefore, not restricted to mediating viral entry but also to modulating biological activity in the recipient host cell. The underlying mechanism of this interaction is not clearly understood, although it has been postulated that the biological relevance of the predominant usage of CCR5 by $\mathrm{V} 3$, in addition to viral transmission, is to ensure that the host cell is at an activated state to facilitate viral replication upon entry $(10,11)$. 
The V3-loop is characterized by its high amino acid variability. It has been proposed that the virus uses this variability as a decoy to evade the host immune responses. The evidence, however, that V3 interacts with host chemokine receptors during viral entry suggests that its role is far more sophisticated. Addressing the question of how a hypervariable domain could induce interactions with specific biological repercussions to the host cell, we showed with immunological $(7-9,12,13)$ and physicochemical methods $(14,15)$ that the degree of $\mathrm{V} 3$-mediated $\mathrm{CD}^{+} \mathrm{T}$ cell activation and subsequent cell death was dependent, at least, on the number of basic amino acids present in the tip of the V3-loop $(6,7,10)$. We tested our hypothesis by constructing synthetic V3 peptides and lipopeptides with appropriate basic amino acid substitutions in the PND to contain 1, 5 or 9 basic residues and a peptide with randomized residues. The in vitro immunological results in primary PBL supported the ionic interaction hypothesis between V3 and CCR5, reinforced by the observation that the relative position of the amino acids in the V3 peptide did not greatly affect the biological phenomenon (13). Surface plasmon resonance analysis of a synthetic 22-mer peptide from the extracellular amino terminal domain of CCR5 (CCR5Nt) in the presence of PND V3 peptides provided direct evidence of the influence of basic amino acids in the CCR5Nt-V3 complex formation $(13,15)$. Sulfation of tyrosines in the CCR5Nt peptide was essential for this interaction in agreement with a previous study (16). Optimal complex formation appeared to occur at an approximate CCR5Nt-V3 2:3 molar ratio. Considering that dimerization/oligomerization of CCR5 triggers intracellular signalling (17), and also the trimeric nature of gp120/gp41 envelope, a potential biological function of the V3-loops may be to facilitate virally-mediated dimerization/oligomerization of CCR5, thus triggering signal transduction in the recipient $\mathrm{CD} 4^{+} \mathrm{T}$ cell.

Computational modeling at atomic resolution has become an integral part of the study of dynamics and thermodynamics of biological systems and processes, once three-dimensional structures are available by traditional diffraction or spectroscopic methods (18). Computational methods can be used to complement and extend further the findings of experimental methods (18). In particular, electrostatic calculations are valuable in the study of the titration properties of proteins and protein complexes and the energetics of recognition and binding (19). Electrostatic calculations have been used successfully to gain insight into the origins of the interactions between viral and immune system proteins and to design mutants with tailored binding and activity properties $(20,21)$. Molecular dynamics simulations and electrostatic calculations in combination with molecular dynamics have also been used to gain theoretical insights into the interaction between HIV-1 gp120 and CD4 $(22,23)$. The recently resolved structure of gp120 (24) allowed us to investigate by electrostatic modeling the interaction of the above-mentioned V3 peptides and the CCR5Nt domain. Fig. 1 shows a ribbon model of the gp120 structure (24). The V3-loop in this structure is a 35-residuelong segment with the sequence $\mathrm{C}^{296}$ TRPNQNTRKSIHIGP GRAFYTTGEIIGDIRQAHC ${ }^{331}$. It protrudes away from the core of gp120, representing an external structure of approximately $50 \AA$ long, $15 \AA$ wide and $5 \AA$ deep, and it is predominantly positively charged. The loop is closed through a disulfide bridge formed between the two end-cysteines. The 'base' is positioned towards the main volume of the protein and contains the disulfide bridge and a short $\beta$-sheet. At the opposite end, the 'tip' contains a flexible $\beta$-hairpin and a $\beta$-turn (24). The region between the base and the tip, called the 'stem' is in random coil conformation. The V3-loop contains 7 basic residues (4 Arg, 2 His, 1 Lys) and two acidic residues (Asp and Glu).

We have postulated that charge-charge interactions drive the interaction between the predominantly positive V3-loop and the predominantly negative $\mathrm{N}$-terminal peptide of CCR5 (6), which contains sulfated tyrosines $(16,25)$. In the present study, we used the coordinates of the gp120 structure (24) to perform structural homology modeling for the same series of V3 peptides with known interacting properties against CCR5Nt (13) and to investigate the role of electrostatics in their potential interaction with the Nt of the whole CCR5.

\section{Materials and methods}

Methods. We used the recently solved crystal structure of HIV-1 gp120 [PDB Code 2B4C; (24)] for our electrostatic modeling. This structure contains gp120 in complex with the CD4 receptor and the X5 antibody, and it differs from earlier structures of gp120 in that it has an intact V3-loop. The apparent flexibility of the V3-loop prevented earlier crystallographic studies from determining its structure. We have used the coordinates of the 35-residue-long V3-loop and 13- and 15-residue segments, which include the V3 tip. The remaining coordinates for gp 120, CD4, X5, and heteroatoms for $\mathrm{N}$-acetyl-D-glucosamine, fucose, sulfate ions, and D-xylitol, which were also present in the structure, were deleted. There are two conformations for the B-turn segment, Gly ${ }^{312}-\mathrm{Pro}^{313}$ Gly $^{314}-\mathrm{Arg}^{315}$, from which we retained the conformation A of the PDB file for our modeling. It must be noted that in the sequence numbering of $2 \mathrm{~B} 4 \mathrm{C}$ there is a numerical (but not structural) gap in the tip (segment $\mathrm{Il}^{309}{ }_{-} \mathrm{Gly}^{312}$ ) and a numerical duplication for residues $\mathrm{Glu}^{322}-\mathrm{Il}^{322}$ in the stem, for sequence alignment purposes (24). Taking these into account the segment between $\mathrm{Cys}^{296}$-Cys $^{331}$ consists of 35 residues. We used 13and 15-residue peptide segments of the V3-loop as structural templates to construct the structures of theoretical mutants of peptides for which there are available binding and activity data against CCR5 $(7,8,12)$. The segment $\mathrm{N}^{302} \mathrm{TRKSIHIGPG}$ $\mathrm{RAFY}^{318}$ was used to construct four 15-residue peptides and the segment $\mathrm{R}^{304} \mathrm{KSIHIGPGRAFY}{ }^{318}$ was used to construct four 13-residue peptides. We used the program Swiss-Pdb Viewer (26) to perform theoretical mutations, based on the structure of the V3-loop of gp120. The best rotamer for each mutated amino acid was automatically selected by the program. In addition, a set of linear structures for these mutant peptides was constructed. The 15-residue peptides were converted to extended conformations by setting all backbone torsion angles to $180^{\circ}$. Several side chain atoms of the extended conformation peptides were found in van der Waals clashes with backbone atoms and some with other side chain atoms. These peptides were subjected to 100 steps of steepest descent energy minimization only on the residues with atoms involved in clashes.

We used a modeled structure of CCR5 to extract the structures of the Nt and extracellular loop domains. This 


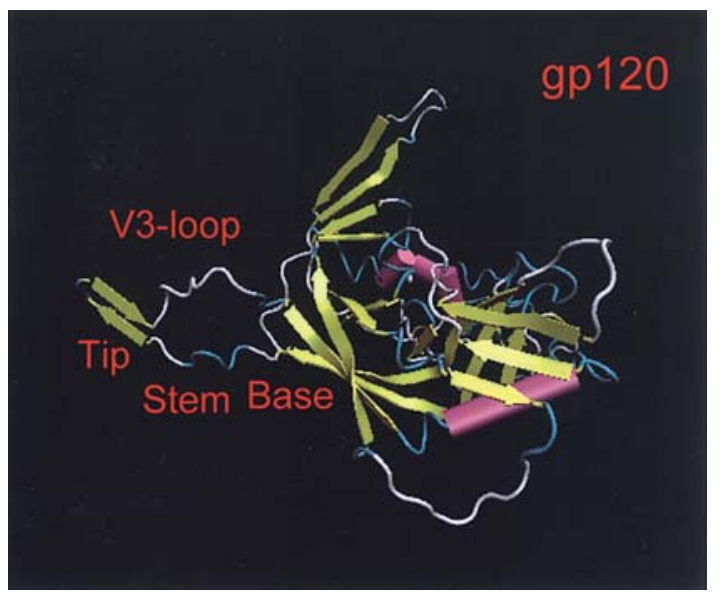

Figure 1. Cartoon representation in a perspective view of the structure of gp120 [PDB Code 2B4C; (24)]. The protruding VCP-loop and its structural components are marked.

structure is one of the 907 putative GPCRs in the human genome, predicted by the Skolnick group using the TASSER method (27); (Code P51681-Model 1 at http://cssb.biology. gatech.edu/skolnick/files/gpcr/gpcr.html).

We used the adaptive Poisson-Boltzmann solver implemented in the program APBS (28) and interfaced with the program VMD (29) to calculate electrostatic potentials. We used the linearized form of the Poisson-Boltzmann equation and a grid of $129 \times 129 \times 129$ points with variable grid lengths depending on the peptides. The parameter set PARSE (30) for partial charges and van der Waals radii was used. The temperature was $298 \mathrm{~K}$, the dielectric coefficients were 78.5 and 4 for the solvent and protein, respectively, and the ionic strength was $150 \mathrm{mM}$, unless otherwise noted. The solvent probe radius was set to $1.4 \AA$ and the mobile ion probe radius was set to $2 \AA$. Histidines were considered neutral for the calculation of electrostatic potentials at physiological $\mathrm{pH}$, unless otherwise noted. Plots of isopotential contours were made at \pm 0.5 or $\pm 1 \mathrm{k}_{\mathrm{B}} \mathrm{T} / \mathrm{e}$, as noted in each figure caption, using VMD (28). Molecular graphics and secondary structure determinations were also prepared using VMD.

\section{Results}

Fig. 2A shows different representations for the backbone and side chains of the V3-loop. Fig. 2B-D shows the spatial distribution of electrostatic potentials in the form of isopotential contour surfaces. Fig. 2B and C shows that the V3-loop is excessively positively charged, owing to +3 charge imbalance between basic (Arg and Lys; His are considered neutral) and acidic residues (Asp and Glu). Plots from calculations with ionic strengths corresponding to the presence of 0 and $150 \mathrm{mM}$ monovalent ions are shown to demonstrate the effect of screening of the electrostatic interactions by salt ions. Maximum intramolecular electrostatic interactions are observed at $0 \mathrm{mM}$ ionic strength, producing overall larger electrostatic potentials (Fig. 2B). We continued our analysis using electrostatic potentials calculated with $150 \mathrm{mM}$ ionic strength as it is closer to physiological conditions. Fig. 2C and D shows plots of isopotential contours at levels of $\pm 0.5 \mathrm{k}_{\mathrm{B}} \mathrm{T} / \mathrm{e}$ and $\pm 1 \mathrm{k}_{\mathrm{B}} \mathrm{T} / \mathrm{e}$ and $150 \mathrm{mM}$ ionic strength, to demonstrate the

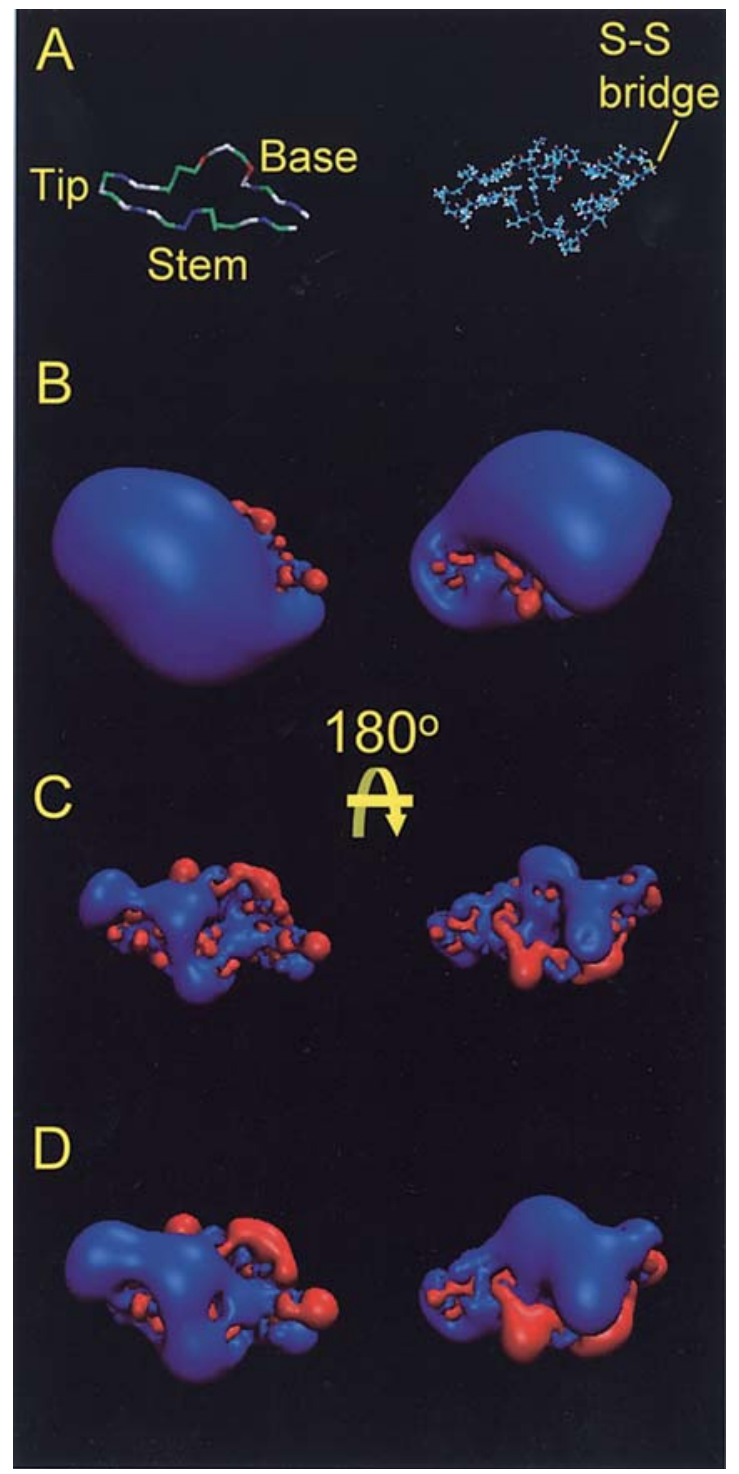

Figure 2. The structure and electrostatic potentials of the isolated V3-loop. (A), $\mathrm{C} \alpha$-trace of the backbone (left) and stick model of the backbone and side chains (right). The color code denotes the following residue types: basic (blue), acidic (red), polar-neutral (green), and non-polar (white). The disulfide bridge is shown in yellow in the right panel. (B), Isopotential contours drawn at $\pm 1 \mathrm{k}_{\mathrm{B}} \mathrm{T} / \mathrm{e}$ from a calculation at $0 \mathrm{mM}$ ionic strength. Blue and red denote positive and negative electrostatic potentials, respectively. The choice of $0 \mathrm{mM}$ ionic strength depicts the maximum intramolecular Coulombic interactions in the absence of attenuation by solvent ions. (C), Isopotential contours drawn at $\pm 1 \mathrm{k}_{\mathrm{B}} \mathrm{T} / \mathrm{e}$ from a calculation at $150 \mathrm{mM}$ ionic strength. (D), Same calculation as (C) (150 mM ionic strength), but the isopotential contours are drawn at $\pm 0.5 \mathrm{k}_{\mathrm{B}} \mathrm{T} / \mathrm{e}$. The right panels correspond to $180^{\circ}$-rotation around the horizontal axis, as marked. The electrostatic potentials were calculated with the backbone Nt amide and $\mathrm{Ct}$ carboxylate charged, as in the case for unblocked free peptides in solution.

effects of these plotting parameters in the visual representations of the electrostatic potentials. In our analysis, we used either \pm 0.5 or $\pm 1 \mathrm{k}_{\mathrm{B}} \mathrm{T} / \mathrm{e}$ for the plots of isopotential contours with the criterion to maximize the electrostatic potential within the calculation grid, but in a self-consistent way for all panels in each figure hereafter.

Fig. 3 shows the structures and electrostatic potentials for the parent and four peptides of the LAI strain. The structure of the parent peptide is derived from the structure of gp120 

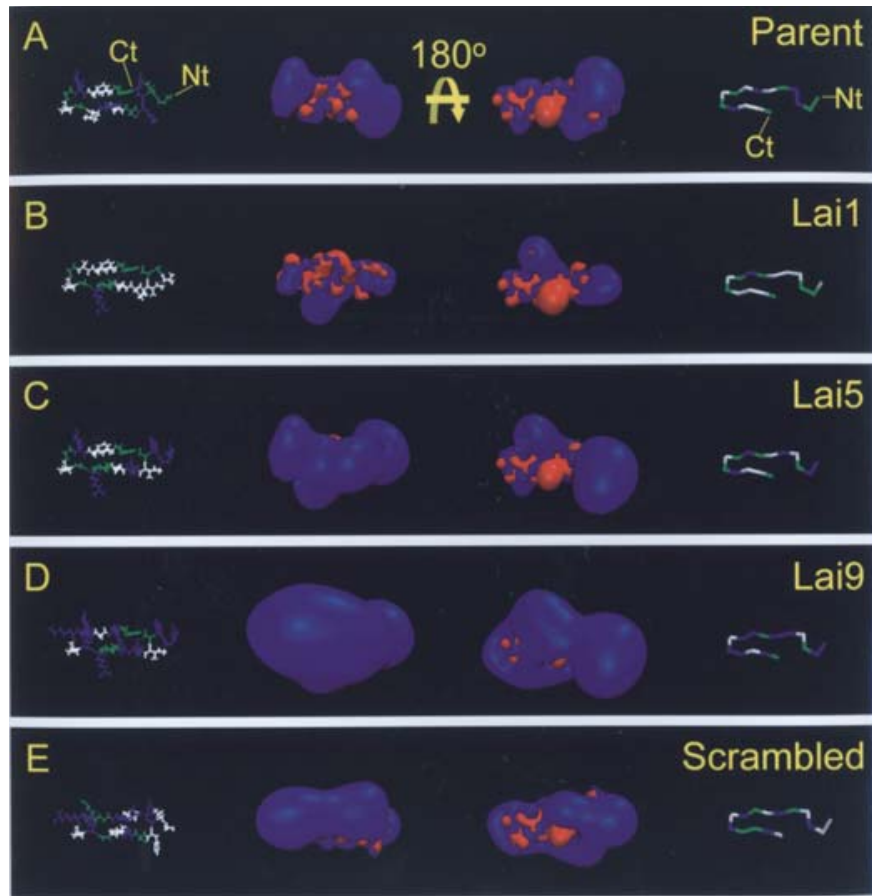

Figure 3. Structures and electrostatic potentials of the 15-residue peptides of Table I. (A), Parent peptide [from the structure with PDB Code 2B4C; (24)]. (B), Lai1, modified Lai strain with total charge +1. (C), Lai5, Lai strain which has total charge +1 . (D), Lai9, Lai strain with total charge +9 . (E), Scrambled, which is Lai of total charge +5 but with scrambled amino acid sequence. The left panels show stick representations of the backbones and side chains and the right panels show $\mathrm{C} \alpha$-trace representations of the backbones. The color code for the left and right panels denotes the following residue types: basic (blue), acidic (red), polar-neutral (green), and non-polar (white). The two middle panels show isopotential contours at $\pm 1 \mathrm{k}_{\mathrm{B}} \mathrm{T} / \mathrm{e}$ from calculations at $150 \mathrm{mM}$ ionic strength. In the middle panels, blue and red denote positive and negative electrostatic potentials, respectively. The two right panels correspond to $180^{\circ}$-rotation around the horizontal axis, as marked. The electrostatic potentials were calculated with the backbone Nt amide and $\mathrm{Ct}$ carboxylate charged, as is appropriate for unblocked free peptides in solution.
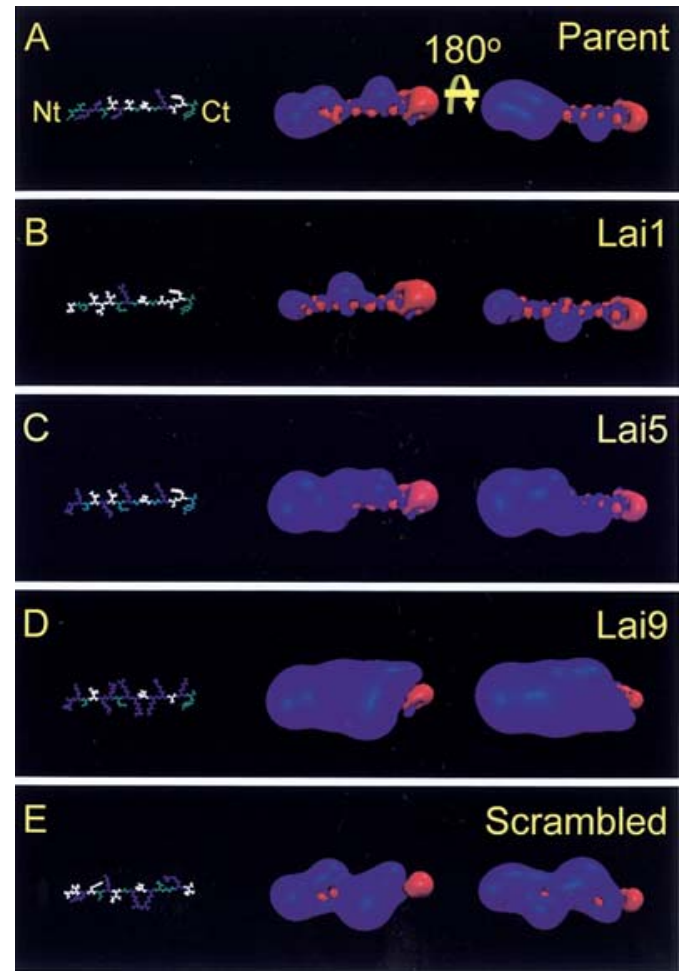

Figure 4. Structures and electrostatic potentials of the 15-residue peptides of Table I in extreme linear conformations. (A), Parent peptide [from the structure with PDB Code 2B4C; (24)]. (B), Lai1, modified Lai strain with total charge +1 . (C), Lai5, Lai strain which has total charge +1 . (D), Lai9, Lai strain with total charge +9 . (E), Scrambled, which is Lai of total charge +5 but with scrambled amino acid sequence. The left panels show stick representations of the backbones and side chains. The color code for the left panels denotes the following residue types: basic (blue), protonated His (cyan), acidic (red), polar-neutral (green), and non-polar (white). The middle and right panels show isopotential contours at $\pm 0.5 \mathrm{k}_{\mathrm{B}} \mathrm{T} / \mathrm{e}$ from calculations at $150 \mathrm{mM}$ ionic strength. In these panels, blue and red denote positive and negative electrostatic potentials, respectively. The right panels correspond to $180^{\circ}$-rotation around the horizontal axis, as marked. The electrostatic potentials were calculated with the backbone Nt amide and Ct carboxylate charged, as is appropriate for unblocked free peptides in solution.

Table I. Peptides studied and their available experimental data.

\begin{tabular}{|c|c|c|c|c|c|c|}
\hline Viral strain & Tropism $^{\mathrm{a}}$ & Amino acid sequence & Charge $^{b}$ & Function $^{c}$ & Binding $^{\mathrm{d}}$ & $\%$ Inhibition $^{\mathrm{e}}$ \\
\hline \multicolumn{7}{|l|}{ 15-residue peptides } \\
\hline 2B4C structure & & NTR KSI HIG PGR AFY & 3 & N/A & N/A & N/A \\
\hline Lai9 & - & RKS IRR QRR PKR AKY & 9 & 42.8 & 786 & 88 \\
\hline Lai5 & $\mathrm{X} 4$ & RKS IRI QRG PGR AFY & 5 & 55.3 & 77 & 75 \\
\hline Lai scrambled & - & AVR FSK IGR PRQ GRI & 5 & 51.5 & 72 & 73 \\
\hline Lai1 & - & VGS ILI QRG PGA AFY & 1 & 3.5 & 3 & 23 \\
\hline \multicolumn{7}{|l|}{ 13-residue peptides } \\
\hline 2B4C structure & & RKS IHI $* *$ G PGR AFY & 3 & N/A & N/A & N/A \\
\hline $\mathrm{Mn}$ & $\mathrm{X} 4$ & RKR IHI $* * G$ PGR AFY & 4 & 20.5 & 69 & 47 \\
\hline Sf128 & $\mathrm{R} 5 \mathrm{X} 4$ & RKS IYI $* * G$ PGR AFY & 3 & 2.4 & 7 & 45 \\
\hline Sf162 & R5 & RKS ITI $* * G$ PGR AFY & 3 & 4.9 & 45 & 45 \\
\hline $\mathrm{Sf} 2$ & $\mathrm{R} 5$ & RKS INI $* * G$ PGR AFY & 3 & 2.7 & 14 & 38 \\
\hline
\end{tabular}

${ }^{\mathrm{a}}$ According to ref. 47. ${ }^{\mathrm{b}}$ The sum of charges for Arg, Lys, Asp, and Glu residues. Histidines are considered neutral at physiological pH 7.4 ${ }^{\mathrm{c} B i o l o g i c a l}$ function measured as relative proliferation (13). ${ }^{\mathrm{d}}$ Relative response with $22 \mathrm{mer}$ CCR5Nt peptide by Surface Plasmon Resonance

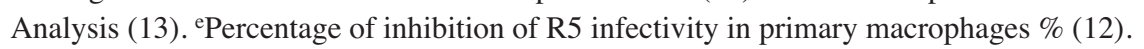


(PDB Code 2B4C) and has been used as a template for the construction of the structures of the LAI peptides, as described in Materials and methods. Table I shows available experimental data for function, binding, and infectivity inhibition of the studied peptides $(7,8,12,13)$. We were interested in the binding data of Table I because they can be directly compared to our electrostatic potential data. When electrostatics drives recognition and binding, there must be a correlation between the strength of the electrostatic potentials and binding affinities. This was the case for the binding affinities of the LAI peptides in the following order: Lai9 > Lai5 Laiscrambled > Lail (Fig. 3 and Table I). Of particular interest is the fact that the Lai5 and Lai-scrambled peptides, both of which possess a +5 excess of charge, also show about the same binding affinities despite the fact that the distribution of positively charged residues is different (Fig. 3 and Table I). This is possibly the case because there is no significant spatial cancellation of positive and negative electrostatic potentials. Negative charge contributions to the electrostatic potentials shown in Fig. 3 originate from the backbone carboxy terminus $\mathrm{COO}^{-}$group and partial charges of electric dipoles.

Because peptides in solution are highly flexible and form ensembles of interconverting conformers, the spatial distributions of electrostatic potentials may be highly fluctuating. We have constructed an additional set of 15-residue peptides of the LAI strain with extended conformations to evaluate the spatial distribution of electrostatic potentials at an extreme linear case. These peptides also account for possible problems from an imprecise sequence alignment at the amino-terminal end of the LAI peptides with the parent peptide (Table I). (The sequence alignment for the 13-residue peptides, shown in Table I, was used to circumvent the issue of the gap for residues 310 and 311, discussed in Materials and methods). Fig. 4 shows the structures and electrostatic potentials of extended peptides. The correlations between the strength of electrostatic potentials and binding affinities is the same as with the structured peptides of Fig. 3 and follows the order: Lai9 > Lai5 Lai-scrambled > Lai1 (Fig. 4 and Table I). The backbone linearity imposes least side chain-side chain and side chain-backbone interactions, which contribute to smaller enhancement or subtraction of electrostatic potentials. This is the reason that we have plotted the isopotential contours in Fig. 4 at $\pm 0.5 k_{\mathrm{B}}$ T/e (as opposed to $\pm 1 \mathrm{k}_{\mathrm{B}}$ T/e of Fig. 3).

Fig. 5 shows the structures and electrostatic potentials for the 13-residue peptides of Table I. Correlations between the strength of the electrostatic potentials of the peptides (Fig. 5) and their binding affinities (Table I) follow the order: $\mathrm{Mn}>$ Sf2 $\sim$ Sf128 Sf162. The spatial distribution of electrostatic potentials suggests a similar effect for the binding affinity of Mn, Lai5, and Lai-scrambled (Figs. 3 and 5), which is supported by the experimental data (Table I). Some subtleties may be present in Mn because of the presence of His308. Fig. 5 also shows that the strength of electrostatic potentials for the parent and Mn peptides is enhanced if the sole His residue is protonated. The His protonation adds an extra +1 charge in Mn and makes it comparable to Lai5 and Laiscrambled (Table I). In our study we have considered His residues to be neutral at physiological $\mathrm{pH}$, because the $\mathrm{pK}_{\mathrm{a}}$ of free His in solution is about 6.3. In order for His to be positively charged at physiological $\mathrm{pH}$, its $\mathrm{pK}_{\mathrm{a}}$ has to be
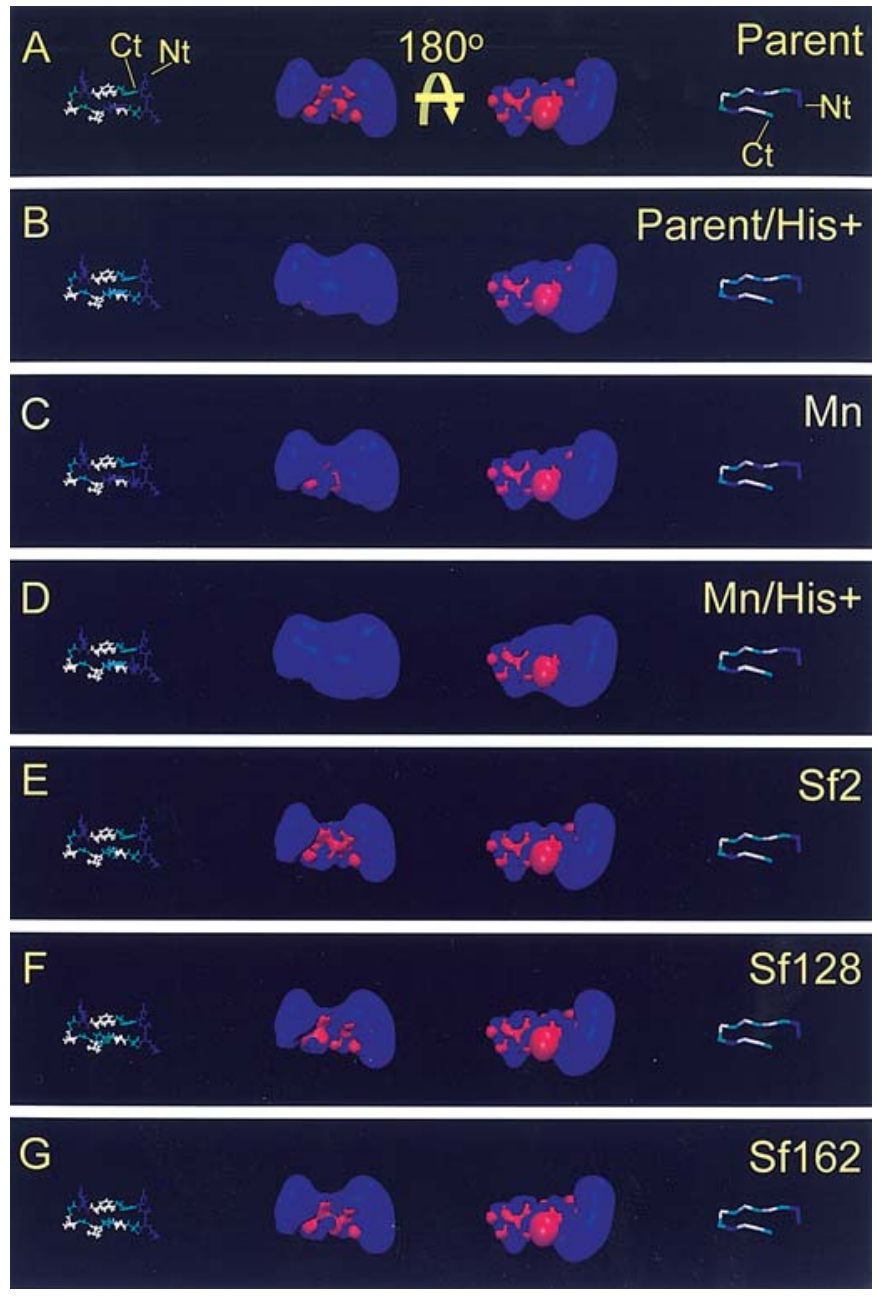

Figure 5. Structures and electrostatic potentials of the 13-residue peptides of Table I. (A), Parent peptide [from the structure with PDB Code 2B4C; (24)]. (B), Parent peptide with the sole His residue protonated. (C), Mn strain. (D), Mn strain with the sole His residue protonated. (E), Sf2 strain. (F), Sf128 strain. (G), Sf162 strain. The left panels show stick representations of the backbones and side chains and the right panels show $\mathrm{C} \alpha$-trace representations of the backbones. The color code for the left and right panels denotes the following residue types: basic (blue), acidic (red), polar-neutral (green), and non-polar (white). The two middle panels show isopotential contours at $\pm 1 \mathrm{k}_{\mathrm{B}} \mathrm{T} / \mathrm{e}$ from calculations at $150 \mathrm{mM}$ ionic strength. In the middle panels, blue and red denote positive and negative electrostatic potentials, respectively. The two right panels correspond to $180^{\circ}$-rotation around the horizontal axis, as marked. The electrostatic potentials were calculated with the backbone $\mathrm{Nt}$ amide and $\mathrm{Ct}$ carboxylate charged, as is appropriate for unblocked free peptides in solution.

elevated to above 7.5. We see no reason for this to happen, unless there is a favorable strong Coulombic interaction with an acidic residue (e.g., Asp or Glu), which are not present in the peptides. The only acidic site is the backbone C-terminal carboxylate, which is located 8-10 $\AA$ away from the pronation sites $\left(\mathrm{N}^{\delta 1}\right.$ and $\mathrm{N}^{\varepsilon 2}$ atoms) of His 308 and suggests a weak Coulombic interaction, if any. Nevertheless, to account for the possibility of a protonated His 308 under experimental conditions, we show the spatial distribution of electrostatic potentials for the parent and Mn peptides when His308 is positively charged (Fig. 5B and D). Protonation of His308 enhances the positive electrostatic potential of the Mn peptide (Fig. 5C and D) but not to the extent of Lai9. 
Table II. Sequences and charges of the extracellular domains of CCR5. Nt and ECL1-3 refer to the N-terminal and the other three extracellular domains.

\begin{tabular}{|c|c|c|c|c|}
\hline Peptide & & Amino acid sequence & & Charge \\
\hline \multicolumn{5}{|c|}{ Sequences from Zhang et al (30) } \\
\hline Nt (Met1-Asn24) & MDY QVS SPI & YDI NYY TSE PCQ & KIN & $-1^{a}$ \\
\hline ECL1 (Ala91-Phe96) & AAQ WDF & & & -1 \\
\hline ECL2 (Phe166-Trp190) & FTR SQK EGL & HYT CSS HFP YSQ & YQF W & 1 \\
\hline ECL3 (Gly265-Leu275) & GLN NCS SSN & RL & & 1 \\
\hline \multicolumn{5}{|c|}{ Sequences from Agrawal et al (31) } \\
\hline Nt (Met1-Arg31) & MDY QVS SPI & YDI NYY TSE PCQ & KIN VKQ IAA R & $1^{\mathrm{a}}$ \\
\hline ECL1 (Tyr89-Gln102) & YAA AQW DFG & NTM CQ & & -1 \\
\hline ECL2 (Ser169-Lys197) & SQK EGL HYT & CSS HFP YSQ YQF & WKN FQT LK & 2 \\
\hline ECL3 (Asn258-Gln280) & NTF QEF FGL & NNC SSS NRL DQA & MQ & -1 \\
\hline
\end{tabular}

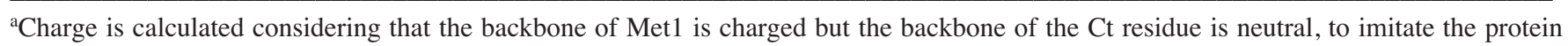
environment. All other peptides in this table are considered uncharged at their termini.

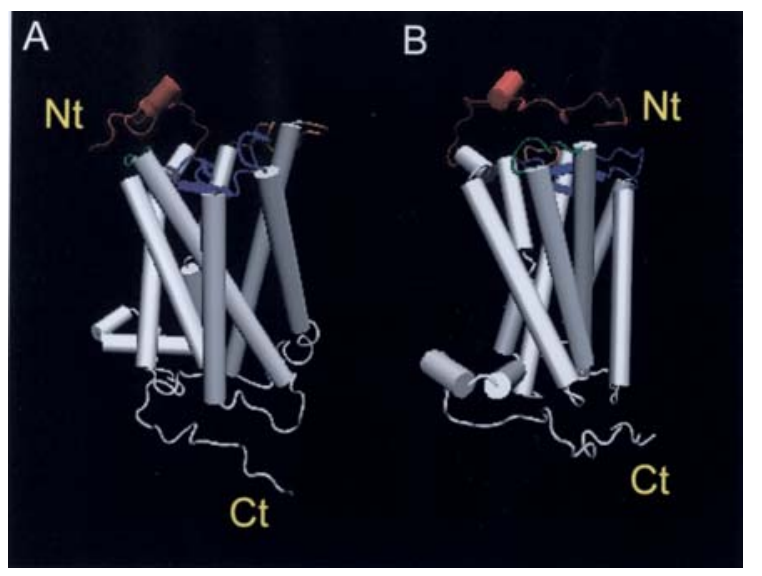

Figure 6. Cartoon representation in a perspective view of the structure of CCR5 (27); Code P51681-Model 1. The extracellular domains are colored as follows: Nt, red; ECL1, green; ECL2, blue; ECL3, orange. The two panels depict different views for the relative topologies of the extracellular domains.

Fig. 6 shows a model of the CCR5 structure [Code P51681-Model 1 (27)] in two different views to demonstrate possible relative orientations of the extracellular domains. The sequences of the extracellular domains are given in Table II. The extracellular domains are predominantly in random coil conformations and we expect them to be flexible. This may be more true for the $\mathrm{Nt}$ domain, as it is anchored to a transmembrane helix only through one of its termini. Indeed, the Nt domain is capable of capping the whole extracellular region of CCR5 (Fig. 6B). The Nt domain has a small portion in an $\alpha$-helical conformation and the ECL2 domain has a small portion in a 3 -sheet conformation (Fig. 6).

Fig. 7 shows the spatial distribution of the electrostatic potentials for the extracellular domains of CCR5, depicted in color in Fig. 6. Overall, the Nt and ECL1 domains are more negative than the ECL2 and ECL3 domains and potentially more amenable to binding with the V3-loop of gp120 or the V3-loop-derived peptides discussed above. The role of ECL1

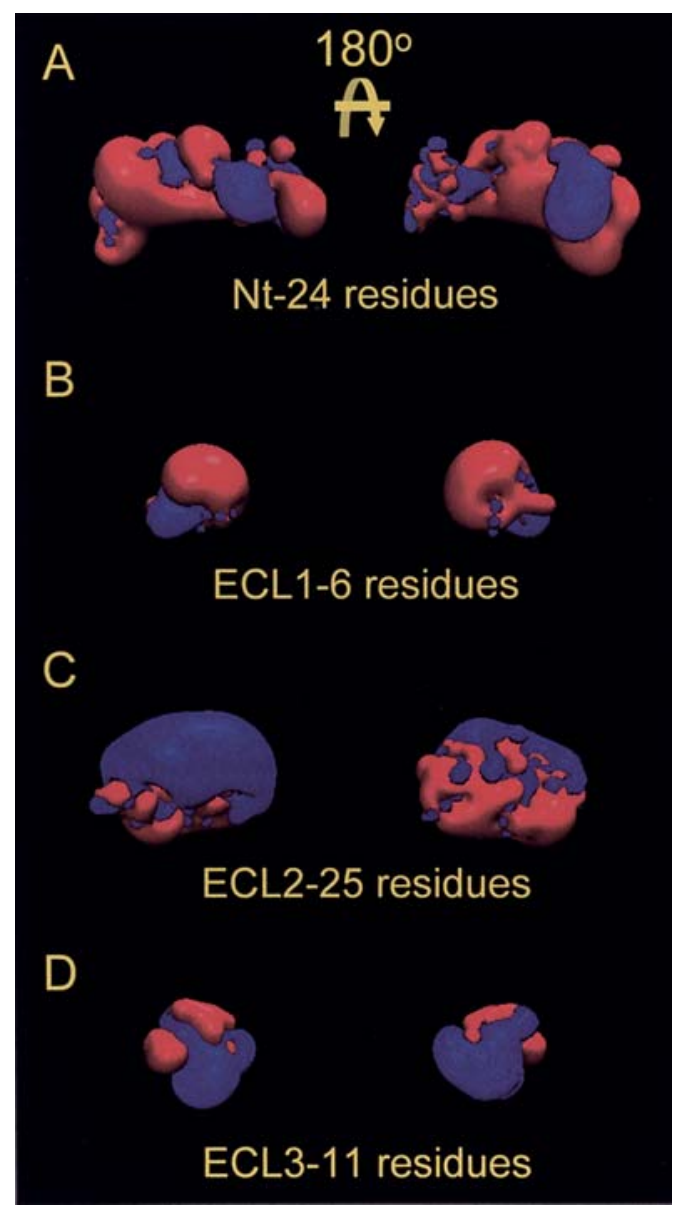

Figure 7. Electrostatic potentials of the extracellular domains of CCR5, from Fig. 6 [sequences from Zhang et al (27)]. (A), Nt. (B), ECL1. (C), ECL2. (D), ECL3. The orientation of the extracellular loops is as in panel (B) of Fig. 6. Isopotential contours are plotted at $\pm 0.5 \mathrm{k}_{\mathrm{B}} \mathrm{T} / \mathrm{e}$ from calculations at $150 \mathrm{mM}$ ionic strength. Blue and red denote positive and negative electrostatic potentials, respectively. The right panels correspond to $180^{\circ}$-rotation around the horizontal axis, as marked. With the exception of $\mathrm{Nt}$, the electrostatic potentials were calculated with the backbone $\mathrm{Nt}$ amide and $\mathrm{Ct}$ carboxylate uncharged, to imitate the peptide bond within the protein environment. For the same reason, the amide of the Nt-domain was kept charged in the calculation. 
may be minor, as it is very short and more rigid than $\mathrm{Nt}$ (it consists of only 6 residues; Table II). The complementarity of the electrostatic potentials of the Nt and ECL2 domains, at least in a particular favourable relative orientation, may be a factor contributing to the stability of the extracellular region when there is no competition from a ligand for entry. The data of Fig. 7 should be interpreted with caution, since the structure is a computational model derived in the absence of the membrane. Additions or truncations of a few residues at the termini of each domain may be sufficient to alter the calculated electrostatic potentials if they contain acidic or basic residues. For comparison, we include in Table II the sequences of the extracellular domains presented in ref. 31. For example, inclusion of 31 residues in the $\mathrm{Nt}$ domain instead of 24 , as proposed in ref. 31 , generates a net charge of +1 (Table II) and predominantly positive potential at the C-terminal base of the Nt domain (data not shown). In our study, we followed structural arguments for the selection of the studied sequences, using the structure of Zhang et al (27). An experimental structure or a model-structure of CCR5 embedded in a membrane and relaxed during a molecular dynamics simulation could have been a possibly more realistic choice to derive the sequences of the extracellular domains.

Overall, the use of spatial distributions of electrostatic potentials is more powerful than sequence arguments alone (e.g., count of charges) because there can be spatial cancellation between positive and negative potentials of neighboring regions. Thus, the electrostatic potentials account for the strength of the interactions. They also account for contributions from partial charges from electric dipoles, which are included in the Poisson-Boltzmann calculations.

\section{Discussion}

Infection of $\mathrm{CD}^{+} \mathrm{T}$ cells for macrophage-tropic HIV-1 strains involves interaction of viral gp120 with host cell CCR5 as a major coreceptor. Crystallographic data have provided useful information on the structures of gp120 bound to CD4, but the corresponding interaction sites between CCR5 and the gp120/ CD4 complex have not been matched as no structure of the CD4/gp120/CCR5 complex has been resolved yet. Although the underlying mechanism for this interaction is not known, mounting evidence suggests the participation of the viral V3loop and the Nt and ECL2 from CCR5.

In the present study, we applied computational modeling methods at atomic resolution and electrostatic calculations to complement our experimental findings for the modulation effects of synthetic linear PND V3-peptides we observed on primary cells. A physicochemical model for protein-protein or protein-ligand association involves a two-step process. The first step entails recognition between the two components and the formation of a non-specific encounter complex. The second step is the specific binding process, which involves the rearrangement of the backbone and side chains in the association interface and exclusion of solvent molecules. The recognition process is driven by long-range electrostatic interactions between protein or peptide macrodipoles, like those shown in Figs. 2-5, 7. Long-range electrostatic interactions are also present during the second step, the binding process. However, the binding step is typically dominated by short- range interactions (electrostatic or not), such as hydrogen bonds, salt bridges, van der Waals, and hydrophobic interactions. Entropy is also a significant contributing factor during the binding step, although more difficult to model.

The studies presented here focus on the recognition step, since we have structures for the putative individual components of the peptide-peptide or protein-peptide complexes but we do not have the structure of the actual complex to derive the detailed physicochemical characteristics of the binding sites. We have investigated possible peptide-peptide interactions between various PND-derived peptides from the V3-loop of gp120 and the N-terminal domain of CCR5. These recognition interactions are possible because of the complementary character of the spatial distribution of the predominantly positive electrostatic potentials of the V3-peptides and the predominantly negative electrostatic potential of the CCR5Nt peptide. Of course, potency depends on the length and amino acid composition of the peptides. The PND V3-peptides cover a small portion of the stem and the whole tip region of the V3-loop. Our results showed that the structure and the electrostatic character of this domain justify active participation in the interaction with the CCR5Nt (Fig. 7). The lack of restricted conformations in the V3-loop that could regulate its interaction with CCR5 and the characteristic electrostatic nature of PND appear to be the dominating features that may influence the observed process of activation-induced cell death, although it is not clear whether this interaction leads to an actual V3CCR5 binding.

By using chimeras consisting of CCR5 domains on a CCR2b background, Rucker et al (32) demonstrated that the $\mathrm{Nt}$ of CCR5 was the only region that, when introduced into CCR2b, conferred M-tropic cofactor activity to the resulting chimera, demonstrating the major role of $\mathrm{Nt}$ in gp120 binding to CCR5. By using amino terminus truncations and alanine substitution mutants, Blanpain et al (33) reported that a number of Nt residues were involved in gp120 binding and coreceptor function. Interestingly, the substitution of amino acids involved in chemokine binding was found to affect gp120 binding and coreceptor activity as well $(33,34)$, postulating that negatively charged residues in the CCR5Nt may directly interact with positively charged residues from the V3-loop (33). The finding that synthetic peptides containing sulfated Y10 and Y14 residues in the CCR5Nt bind gp120 at micromolar affinities provided further evidence for the direct involvement of the CCR5Nt domain with gp120 (35). Posttranslational sulfation of Y10 and Y14, therefore, plays a role in both ligand binding and coreceptor activity $(35,36)$. Navenot et al $(37)$ reported that amino acid residues in ECL2 are involved in coreceptor activity of CCR5 with M-tropic envelope glycoproteins, whereas the Nt of CCR5 does not determine the specificity of chemokine binding, thus concluding that the molecular anatomy of CCR5 binding is critical in antagonism of coreceptor activity.

Liu et al (38) have generated structural models of CCR5 in complex with gp120 and CD4 through protein structure modeling, docking and molecular dynamics simulation. On the basis of their models, the binding of CD4 to gp120 induces a conformational change in gp120 and exposes the binding site for CCR5. The extended acidic Nt region in CCR5 interacts firstly with the basic bridging sheet in gp120 inducing 
conformational changes in the Nt thus leading the V3-loop region close to the ECL2 domain.

Hsu et al $(22,23)$ performed molecular dynamics simulations in explicit solvent based on the gp120/CD4/CD4i crystal structure in conjunction with modeled V3 and V4 loops to gain insight into the dynamics of the binding process. Three differentiated interaction modes between CD4 and gp120 were found, which involve electrostatics, hydrogen bond and van der Waals networks. The abundant basic residues of the V3-loop (six arginines and two lysines) are thought to facilitate the recruitment of the generally acidic chemokine coreceptor subsequent to the binding of CD4 (45-47). They generated a positive electrostatic potential that occupied a large space centered at the basal region of the V3-loop. Moving from the free to the complex form, they relocated the positive potential towards the coreceptor binding site.

Emerging data on the structures of the coreceptors indicate that the regions identified as being important for binding gp120 - the coreceptor Nt and ECL2 - may also be spatially separated (39). By integrating the two-site gp120 binding site on the coreceptor with the two-site coreceptor binding site that we observe in the core V3 gp120 structure, Kwong et al (40) proposed that the $\mathrm{Nt}$ of the coreceptor reaches up and binds to the core and V3 base while the V3 tip reaches down to interact with the ECL2 of the coreceptor. The positive electrostatic character of the V3 peptide is apparent, and the crown of the tip is more positive than the bottom of the tip; the bottom of the stem is more positive than the top of the stem; and finally, the bottom of the base is more positive than the top of the base. It is interesting that Kwong et al (40) suggested that the sulfated negative CCR5Nt (4 Tyr) interacts with the base rather than the stem and/or tip as it is more positive and therefore more suitable for this interaction.

Seibert et al (41) has carried out a targeted mutagenesis screen to define the amino acid residues in CCR5 that form a ligand binding pocket for a series of small-molecule inhibitors of cellular HIV-1 entry. It was shown that small inhibitory molecules bind to a pocket in the transmembrane domain of CCR5 and non-competitively block gp120 binding to the coreceptor. However, these molecules failed to inhibit the gp120 interaction with CCR5Nt, whereas they blocked binding of several monoclonal antibodies that recognize epitopes in the second extracellular loop of CCR5. Safarian et al (42) further explored the inhibition of HIV-1 entry by the two small molecules and an anti-CCR5 MAb. Sensitivity to the small molecules was dependent on gp120 affinity for CCR5, and this was regulated by the V3-loop crown. In their proposed model the crown of the V3-loop interacts with ECL2 of CCR5, thereby determining the efficiency of entry inhibition.

The above evidence indicates that both the Nt and ECL2 domains in CCR5 are critical for viral envelope binding and fusion mediated by the V3-loop. It is likely that ECL2 and the surrounding hydrophobic transmembrane domains actively participate in the gp120 specific binding and fusion steps. However, it is far from clear which part of the V3-loop interacts with which extracellular domain of CCR5, as the evidence to date is conflicting $(38,42,43)$. We have investigated possible peptide-protein non-specific recognition interactions between the various peptides derived from the V3-loop of gp120 and the whole CCR5. In particular, we focused on the Nt and ECL2 extracellular domains of CCR5. In a coarse comparison, it appears that the $\mathrm{Nt}$ domain is more amenable to $\mathrm{V} 3$ peptide binding, than ECL2, because of domination of negative electrostatic potential. However, there are positive patches in $\mathrm{Nt}$ and negative patches in ECL2 (Fig. 7), which upon binding and the associated structural rearrangements may be contributing factors to the stabilization of the complex. A sequential twostep specific binding, following the non-specific recognition, may also be possible, involving different extracellular domains. The specifics of the electrostatic potentials may play a role in the previously proposed interaction mode, e.g., 2:3 ratio in CCR5:V3-peptide. However, we cannot derive a more specific model in the absence of a three-dimensional structure of such a complex.

Activation of the target $\mathrm{T}$ cell and binding of gp120 are probably two distinct functions of the V3-loop (44-46). In the model we have proposed $(6,10)$, the PND domain preferentially interacts with the CCR5Nt to deliver an additional signal to TcR-mediated pathway (9). The present electrostatic calculations and computational modeling on the V3-loop support this notion.

\section{Acknowledgments}

The project was financially supported by the General Secretariat for Research and Technology, Greece, grant 4.5.1-SP-YB/31 (2003) and partially by NSF (D.M.).

\section{References}

1. Wei X, Ghosh SK, Taylor ME, et al: Viral dynamics in human immunodeficiency virus type 1 infection. Nature 373: 117-122, 1995.

2. Whitaker RE: Re-assessing the virological approach to HIV pathogenesis: can it explain AIDS as an immunological disease? J Theor Biol 187: 45-56, 1997.

3. Anderson RW, Ascher MS and Sheppard HW: Direct HIV cytopathicity cannot account for CD4 decline in AIDS in the presence of homeostasis: a worst-case dynamic analysis. J Acquir Immune Defic Syndr Hum Retrovirol 17: 245-252, 1998 .

4. Douek DC, Picker LJ and Koup RA: T cell dynamics in HIV-1 infection. Annu Rev Immunol 21: 265-304, 2003.

5. Berger EA, Doms RW, Fenyo EM, et al: A new classification for HIV-1. Nature 391: 240, 1998.

6. Krambovitis E and Spandidos DA: HIV-1 infection: is it time to reconsider our concepts? Int J Mol Med 18: 3-8, 2006.

7. Zafiropoulos A, Baritaki S, Sioumpara M, Spandidos DA and Krambovitis E: V3 induces in human normal cell populations an accelerated macrophage-mediated proliferation - apoptosis phenomenon of effector $\mathrm{T}$ cells when they respond to their cognate antigen. Biochem Biophys Res Commun 281: 63-70, 2001 .

8. Zafiropoulos A, Baritaki S, Vlata Z, Spandidos DA and Krambovitis E: Dys-regulation of effector $\mathrm{CD}^{+} \mathrm{T}$ cell function by the V3 domain of the HIV-1 gp120 during antigen presentation. Biochem Biophys Res Commun 284: 875-879, 2001.

9. Porichis F, Vlata Z, Hatzidakis G, Spandidos DA and Krambovitis E: HIV-1 gp120/V3-derived epitopes promote activation-induced cell death to superantigen-stimulated CD4(+)/ CD45RO(+) T cells. Immunol Lett 108: 97-102, 2007.

10. Krambovitis E, Zafiropoulos A, Baritaki S and Spandidos DA: Simple electrostatic interaction mechanisms in the service of HIV-1 pathogenesis. Scand J Immunol 59: 231-234, 2004.

11. Krambovitis E, Porichis F and Spandidos DA: HIV entry inhibitors: a new generation of antiretroviral drugs. Acta Pharmacol Sin 26: 1165-1173, 2005.

12. Baritaki S, Dittmar MT, Spandidos DA and Krambovitis E: In vitro inhibition of R5 HIV-1 infectivity by X4 V3-derived synthetic peptides. Int J Mol Med 16: 333-336, 2005. 
13. Baritaki S, Zafiropoulos A, Sioumpara M, Politis M, Spandidos DA and Krambovitis E: Ionic interaction of the HIV-1 V3 domain with CCR5 and deregulation of T lymphocyte function. Biochem Biophys Res Commun 298: 574-580, 2002.

14. Galanakis PA, Spyroulias GA, Rizos A, Samolis P and Krambovitis E: Conformational properties of HIV-1 gp120/V3 immunogenic domains. Curr Med Chem 12: 1551-1568, 2005.

15. Rizos AK, Morikis D, Galanakis P, Spyroulias GA and Krambovitis E: Characterization of the interaction between peptides derived from the gp120/V3 domain of HIV-1 and the amino terminal of the chemokine receptor CCR 5 by NMR spectroscopy and light scattering. J Non-Crystalline Solids 352: 4451-4458, 2006.

16. Farzan M, Mirzabekov T, Kolchinsky P, et al: Tyrosine sulfation of the amino terminus of CCR5 facilitates HIV-1 entry. Cell 96: 667-676, 1999

17. Wong MM and Fish EN: Chemokines: attractive mediators of the immune response. Semin Immunol 15: 5-14, 2003.

18. Morikis D and Lambris JD: Physical methods for structure, dynamics and binding in immunological research. Trends Immunol 25: 700-707, 2004

19. Wu J and Morikis D: Molecular thermodynamics for charged biomacromolecules. Fluid Phase Equilibria 241: 317-333, 2006.

20. Sfyroera G, Katragadda M, Morikis D, Isaacs SN and Lambris JD: Electrostatic modeling predicts the activities of orthopoxvirus complement control proteins. J Immunol 174: 2143-2151, 2005.

21. Zhang L and Morikis D: Immunophysical properties and prediction of activities for vaccinia virus complement control protein and smallpox inhibitor of complement enzymes using molecular dynamics and electrostatics. Biophys J 90: 3106-3119, 2006.

22. Hsu ST and Bonvin AM: Atomic insight into the CD4 bindinginduced conformational changes in HIV-1 gp120. Proteins 55: 582-593, 2004

23. Hsu ST, Peter C, van Gunsteren WF and Bonvin AM: Entropy calculation of HIV-1 Env gp120, its receptor CD4, and their complex: an analysis of configurational entropy changes upon complexation. Biophys J 88: 15-24, 2005.

24. Huang CC, Tang M, Zhang MY, et al: Structure of a V3containing HIV-1 gp120 core. Science 310: 1025-1028, 2005.

25. Choe H, Li W, Wright PL, et al: Tyrosine sulfation of human antibodies contributes to recognition of the CCR5 binding region of HIV-1 gp120. Cell 114: 161-170, 2003.

26. Guex N and Peitsch MC: SWISS-MODEL and the SwissPdbViewer: an environment for comparative protein modeling. Electrophoresis 18: 2714-2723, 1997.

27. Zhang Y, Devries ME and Skolnick J: Structure modeling of all identified $\mathrm{G}$ protein-coupled receptors in the human genome. PLoS Comput Biol 2: 88-99, 2006.

28. Baker NA, Sept D, Joseph S, Holst MJ and McCammon JA: Electrostatics of nanosystems: application to microtubules and the ribosome. Proc Natl Acad Sci USA 98: 10037-10041, 2001.

29. Humphrey W, Dalke A and Schulten K: VMD: visual molecular dynamics. J Mol Graph 14: 33-37, 1996.

30. Sitkoff D, Sharp KA and Honig B: Accurate calculation of hydration free energies using macroscopic solvent models. J Phys Chem 98: 1978-1988, 1994.

31. Agrawal L, Vanhorn-Ali Z, Berger EA and Alkhatib G: Specific inhibition of HIV-1 coreceptor activity by synthetic peptides corresponding to the predicted extracellular loops of CCR5 Blood 103: 1211-1217, 2004.

32. Rucker J, Samson M, Doranz BJ, et al: Regions in beta-chemokine receptors CCR5 and CCR2b that determine HIV-1 cofactor specificity. Cell 87: 437-446, 1996.
33. Blanpain C, Doranz BJ, Vakili J, et al: Multiple charged and aromatic residues in CCR5 amino-terminal domain are involved in high affinity binding of both chemokines and HIV-1 Env protein. J Biol Chem 274: 34719-34727, 1999.

34. Farzan M, Choe H, Vaca L, et al: A tyrosine-rich region in the $\mathrm{N}$ terminus of CCR5 is important for human immunodeficiency virus type 1 entry and mediates an association between gp120 and CCR5. J Virol 72: 1160-1164, 1998.

35. Farzan M, Vasilieva N, Schnitzler CE, et al: A tyrosine-sulfated peptide based on the $\mathrm{N}$ terminus of CCR5 interacts with a CD4 enhanced epitope of the HIV-1 gp120 envelope glycoprotein and inhibits HIV-1 entry. J Biol Chem 275: 33516-33521, 2000 .

36. Dorfman T, Moore MJ, Guth $\mathrm{AC}$, Choe $\mathrm{H}$ and Farzan $\mathrm{M}$ : A tyrosine-sulfated peptide derived from the heavy-chain CDR3 region of an HIV-1-neutralizing antibody binds gp120 and inhibits HIV-1 infection. J Biol Chem 281: 28529-28535, 2006.

37. Navenot JM, Wang ZX, Trent JO, et al: Molecular anatomy of CCR5 engagement by physiologic and viral chemokines and HIV-1 envelope glycoproteins: differences in primary structural requirements for RANTES, MIP-1 alpha, and vMIP-II binding. J Mol Biol 313: 1181-1193, 2001.

38. Liu S, Fan S and Sun Z: Structural and functional characterization of the human CCR5 receptor in complex with HIV gp120 envelope glycoprotein and CD4 receptor by molecular modeling studies. J Mol Model (Online) 9: 329-336, 2003.

39. Klco JM, Wiegand CB, Narzinski K and Baranski TJ: Essential role for the second extracellular loop in C5a receptor activation. Nat Struct Mol Biol 12: 320-326, 2005.

40. Kwong PD, Wyatt R, Sattentau QJ, Sodroski J and Hendrickson WA: Oligomeric modeling and electrostatic analysis of the gp120 envelope glycoprotein of human immunodeficiency virus. J Virol 74: 1961-1972, 2000

41. Seibert C, Ying W, Gavrilov S, et al: Interaction of small molecule inhibitors of HIV-1 entry with CCR5. Virology 349: 41-54, 2006

42. Safarian D, Carnec X, Tsamis F, Kajumo F and Dragic T: An anti-CCR5 monoclonal antibody and small molecule CCR5 antagonists synergize by inhibiting different stages of human immunodeficiency virus type 1 entry. Virology 352: 477-484, 2006.

43. Suphaphiphat P, Essex M and Lee TH: Mutations in the V3 stem versus the V3 crown and $\mathrm{C} 4$ region have different effects on the binding and fusion steps of human immunodeficiency virus type 1 gp120 interaction with the CCR 5 coreceptor. Virology (In press)

44. Atchison RE, Gosling J, Monteclaro FS, Franci C, Digilio L, Charo IF and Goldsmith MA: Multiple extracellular elements of CCR5 and HIV-1 entry: dissociation from response to chemokines. Science 274: 1924-1926, 1996.

45. Gosling J, Monteclaro FS, Atchison RE, Arai H, Tsou CL Goldsmith MA and Charo IF: Molecular uncoupling of C-C chemokine receptor 5-induced chemotaxis and signal transduction from HIV-1 coreceptor activity. Proc Natl Acad Sci USA 94: 5061-5066, 1997.

46. Cicala C, Arthos J, Censoplano N, et al: HIV-1 gp120 induces NFAT nuclear translocation in resting $\mathrm{CD}^{+}{ }^{-} \mathrm{T}$-cells. Virology 345: 105-114, 2006

47. Hung CS, van der Heyden N and Ratner L: Analysis of the critical domain in the V3 loop of human immunodeficiency virus type 1 gp120 involved in CCR5 utilization. J Virol 73: 8216-8226, 1999 